\title{
THE ESSENTIAL PART OF A SURFACE
}

PAUL V. REICHELDERFER

This paper attempts to place the concept of the essential part of the projection of a continuous surface upon a plane in its historical and mathematical setting, and to outline the role this concept is playing in the solution of the area problem. To that purpose those events most closely related to this concept will be sketched, and less relevant facts will be suppressed. ${ }^{1}$

1. The length of a curve. Consider a continuous path curve $C$ given by a representation

$$
C: \quad x=x(u), y=y(u), z=z(u), \quad 0 \leqq u \leqq 1,
$$

where the functions $x(u), y(u), z(u)$ are defined, single-valued, realvalued, and continuous on the closed unit interval $0 \leqq u \leqq 1$. It is well known that the length $L(C)$ of $C$ may be defined as the limit of the lengths of inscribed polygons which converge to $C$-if $0=u_{0}<u_{1}$ $<\cdots<u_{i}<\cdots<u_{n}=1$ be a subdivision of $0 \leqq u \leqq 1$, then

$$
\begin{aligned}
L(C)=\lim _{n \rightarrow \infty} \sum_{i=1}^{n}\left\{\left[x\left(u_{i}\right)-x\left(u_{i-1}\right)\right]^{2}+\right. & {\left[y\left(u_{i}\right)-y\left(u_{i-1}\right)\right]^{2} } \\
+ & {\left.\left[z\left(u_{i}\right)-z\left(u_{i-1}\right)\right]^{2}\right\}^{1 / 2}, }
\end{aligned}
$$

the limit ${ }^{2}$ being taken with respect to subdivisions of $0 \leqq u \leqq 1$ for which the maximum value of $\left|u_{i}-u_{i-1}\right|$ for $i$ between 1 and $n$ converges to zero with $1 / n$. Observe that (1.2) gives an expression for the length of the curve $C$ in terms of its representation (1.1), standard algebraic operations, and one limit process.

2. The area of a surface. Consider a continuous path surface $S$

An address delivered before the Ames meeting of the Society on November 30, 1946, by invitation of the Committee to Select Hour Speakers for Western Sectional Meetings; received by the editors November 20, 1946.

1 A list of papers closely related to the facts to be presented in the sequel is included at the end of this paper. Numbers in brackets refer to this bibliography. For an exhaustive treatment of the concepts of length and area and an extensive bibliography upon the subject, the reader should consult Rad6 [4]. This volume of the Colloquium publications is now in the process of being published. The writer had the privilege of reading the manuscript.

2 Of course, it is necessary to discuss the existence of this limit and its independence of the representation chosen for $C$. See Rad6 [4, III. 3]. 
given by a representation ${ }^{8}$

$$
S: \quad x=x(u, v), y=y(u, v), z=z(u, v), \quad 0 \leqq u, v \leqq 1,
$$

where the functions $x(u, v), y(u, v), z(u, v)$ are defined, single-valued, real-valued, and continuous on the closed unit square $0 \leqq u, v \leqq 1$. Before the twentieth century mathematicians generally defined the area of $S$ in a way analogous to that for the length of a curve-namely, as the limit of the areas of inscribed polyhedra which converge to $S$. In $1880 \mathrm{H}$. A. Schwarz inscribed sequences of polyhedra in a right circular cylinder to show these interesting facts (see Schwarz [1]): there are sequences of polyhedra inscribed in the cylinder and converging to it whose areas have no limit; if $r$ be any real number not less than the accepted value for the area of the surface of the cylinder, there are sequences of polyhedra inscribed in the cylinder and converging to it whose areas converge to $r$; in particular there are sequences of polyhedra inscribed in the cylinder and converging to it whose areas converge to plus infinity.

3. The Lebesgue area. Stimulated by Schwarz's discovery, many mathematicians, including Peano, Lebesgue, and Geöcze (see Radó $[2,4])$, set forth definitions for the area of a continuous surface. Lebesgue's definition has gained wide acceptance, and is used in the sequel (see Lebesgue [1]). Given a continuous path surface $S$ as in (2.1), consider a sequence of polyhedra $P_{n}$-not necessarily inscribed in $S$-which converge to $S$. If $E\left(P_{n}\right)$ denotes the elementary area of the polyhedron $P_{n}$-that is, the sum of the areas of its triangular faces-then $\lim \inf E\left(P_{n}\right)$ is an upper bound for the Lebesgue area $L(S)$ of $S$, and $L(S)$ is defined to be the greatest lower bound of all the upper bounds obtained in this way. ${ }^{4}$

4. The area problem. The reader's attention is invited to the following facts: (i) Given a continuous path curve $C$ as in (1.1), consider a sequence of polygons $p_{n}$-not necessarily inscribed in $C$ - which converge to $C$. If $e\left(p_{n}\right)$ denotes the elementary length of the polygon $p_{n}$ - that is, the sum of the lengths of its linear segments-then $\lim \inf e\left(p_{n}\right)$ is an upper bound for the length $L(C)$ of $C$, and $L(C)$ is the greatest lower bound of all the upper bounds obtained in this way (see Radó [4, III. 3]). Thus Lebesgue's definition for the area of a surface is analogous to a possible definition for the length of a curve.

\footnotetext{
3 For a thorough discussion of the representations for continuous curves and surfaces, see Rad6 [4, II. 3].

${ }^{4}$ Again, the independence of $L(S)$ upon the representation chosen for $S$ must be discussed. See Radó [4, V. 2].
} 
(ii) The polyhedron $P_{n}$ is not required to be inscribed in the surface $S$; this permits one to prove that the Lebesgue area $L(S)$ is a lower semicontinuous functional of $S$ - that is, if $S_{n}$ be any sequence of continuous path surfaces converging ${ }^{5}$ to $S$, then $\lim \inf L\left(S_{n}\right)$ is an upper bound for $L(S)$. (iii) In all cases where an area for a given surface has been assigned and widely accepted, the Lebesgue area agrees with the accepted value-in particular, the Lebesgue area $L(P)$ of a polyhedron $P$ is equal to its elementary area $E(P)$. (iv) While the definition of the Lebesgue area $L(S)$ of a continuous surface $S$ guarantees the existence of a sequence of polyhedra $P_{n}$ such that the $P_{n}$ converge to $S$ and the elementary areas $E\left(P_{n}\right)$ converge to $L(S)$, it gives no clue as to a way of constructing such a sequence. (v) Let there be assigned in any manner whatsoever a real number $A(S)$ to every continuous path surface $S$ so that the following three conditions are fulfilled: $A(S)$ is a lower semi-continuous functional of $S$; for every polyhedron, $P, A(P)$ has the same value as the elementary area $E(P)$; for every continuous surface $S$ there exists a sequence of polyhedra $P_{n}$ such that the $P_{n}$ converge to $S$ and the $A\left(P_{n}\right)$ converge to $A(S)$. Then for every continuous surface $S, A(S)$ must have the same value as the Lebesgue area $L(S)$. (vi) The Lebesgue area of a surface $S$ is not directly expressible in terms of its representation (2.1) using standard operations (cf. §1). Hence one of the outstanding problems is the following:

Area Problem: Given a continuous path surface $S$ as in (2.1), express its area in terms of its representation (2.1), using standard operations.

5. A solution to the area problem for a non-parametric surface. In one important special case-the so-called nonparametric case- the area problem is solved. Suppose the continuous path surface $S$ has a representation of the form (see Radó $[4, \mathrm{~V} .3]$ )

$$
S: \quad x=u, y=v, z=z(u, v), \quad 0 \leqq u, v \leqq 1 .
$$

Under no additional hypotheses, Radó has shown (see Radó [1]) that $L(S)$ may be given in terms of the representation (5.1) and standard operations as follows. Consider any sequence of subdivisions $D_{n}$ of the unit square $0 \leqq u, v \leqq 1$ into oriented rectangles $r: u^{\prime} \leqq u \leqq u^{\prime \prime}$, $v^{\prime} \leqq v \leqq v^{\prime \prime}$ for which the maximum of the diameters of the rectangles

- A sequence of continuous surfaces $S_{n}$ converges to a continuous surface $S$ given as in (2.1) if the $S_{n}$ admit of representations $S_{n}: x=x_{n}(u, v), y=y_{n}(u, v), z=z_{n}(u, v)$, $0 \leqq u, v \leqq 1, n=1,2, \cdots$, such that $x_{n}, y_{n}, z_{n}$ converge uniformly on $0 \leqq u, v \leqq 1$ to $x, y, z$ respectively. For a treatment of the concept of convergence for continuous curves and surfaces, see Rad6 [4, II. 3]. 
in $D_{n}$ converges to zero with $1 / n$. Then ${ }^{6}$

$$
\begin{aligned}
L(s)= & \lim _{n \rightarrow \infty} \sum_{r \in D_{n}}\left\{\left[\int_{v^{\prime}}^{v^{\prime \prime}}\left|z\left(u^{\prime \prime}, v\right)-z\left(u^{\prime}, v\right)\right| d v\right]^{2}\right. \\
& +\left[\int_{u^{\prime}}^{u^{\prime \prime}}\left|z\left(u, v^{\prime \prime}\right)-z\left(u, v^{\prime}\right)\right| d u\right]^{2} \\
& \left.+\left[\left(u^{\prime \prime}-u^{\prime}\right)\left(v^{\prime \prime}-v^{\prime}\right)\right]^{2}\right\}^{1 / 2} .
\end{aligned}
$$

6. An expression for the length of a curve. One approach to the area problem in case $S$ is any continuous path surface is to investigate various possible expressions for the length of a curve, expecting that these may afford a clue to a method for attacking the area problem. An expression for the length of a curve which has been especially fruitful will now be considered (see Banach [1]). Let $C$ be a continuous path curve given by the representation (1.1). The projection of $C$ on the $x$-axis is then represented by $x=x(u), 0 \leqq u \leqq 1$. For each interval $i: u^{\prime} \leqq u \leqq u^{\prime \prime}$, where $0 \leqq u^{\prime}<u^{\prime \prime} \leqq 1$, and for each point $x_{0}$, let $N\left(x_{0}, i\right)$ denote the number-possibly zero, possibly plus infinity -of points $u_{0}$ in $i$ such that $x\left(u_{0}\right)=x_{0}$. Then for each interval $i$, $N(x, i)$ is a non-negative measurable function of $x$ which is zero outside of a certain interval, and ${ }^{7} \int N(x, i)$ may be regarded as the length of the one-dimensional curve represented by $x=x(u), u^{\prime} \leqq u \leqq u^{\prime \prime}-$ in fact, $\int N(x, i)$ is equal to the total variation of $x$ over the interval $i$ (see Radó [4, III. 2]). Functions $N(y, i), N(z, i)$ are defined similarly and have analogous properties. Now let $D_{n}$ be any sequence of subdivisions of the interval $0 \leqq u \leqq 1$ into intervals $i: u^{\prime} \leqq u \leqq u^{\prime \prime}$ for which the maximum of the lengths of the intervals in $D_{n}$ converges to zero with $1 / n$. Then ${ }^{8}$

$$
\begin{gathered}
L(C)=\lim _{n \rightarrow \infty} \sum_{i \in D_{n}}\left\{\left[\int N(x, i)\right]^{2}+\left[\int N(y, i)\right]^{2}\right. \\
\left.+\left[\int N(z, i)\right]^{2}\right\}^{1 / 2} .
\end{gathered}
$$

${ }^{6}$ For other possible solutions of the area problem in the nonparametric case, see $\operatorname{Rad} 6$ [4, V. 3].

${ }^{7}$ Let $I$ be any interval on the $x$-axis such that $N(x, i)$ is zero for $x$ not in $I$. If $N(x, i)$ is summable on $I$, then $\int N(x, i)$ denotes the Lebesgue integral of $N(x, i)$ on $I$; if $N(x, i)$ is not summable on $I$, then $\int N(x, i)$ is defined to be plus infinity. Clearly $\int N(x, i)$ is independent of the choice of $I$, so long as $N(x, i)$ is zero for $x$ not in $I$.

${ }^{8}$ For other possible expressions for $L(C)$ see Rad6 [4, III. 3]. 
For purposes of comparison, another formula for $L(C)$ is presented. If $D$ be the generic notation for a subdivision of the interval $0 \leqq u \leqq 1$, then

$$
\begin{gathered}
L(C)=\text { l.u.b. } \sum_{i \in D}\left\{\left[\int N(x, i)\right]^{2}+\left[\int N(y, i)\right]^{2}\right. \\
\left.+\left[\int N(z, i)\right]^{2}\right\}^{1 / 2},
\end{gathered}
$$

the least upper bound being taken with respect to all subdivisions $D$. Formula (6.1) is preferable to (6.2) because it states that $L(C)$ may be computed as the limit of sums over any sequence of subdivisions $D_{n}$ of $0 \leqq u \leqq 1$ for which the maximum of the lengths of the intervals in $D_{n}$ converges to zero with $1 / n$.

7. An expression for the area of a surface. Now let $S$ be a continuous path surface given by the representation (2.1). The projection of $S$ on the $x y$-plane is then represented by

$$
x=x(u, v), \quad y=y(u, v), \quad 0 \leqq u, v \leqq 1 .
$$

For each set $E$ in $0 \leqq u, v \leqq 1$, and for each point $\left(x_{0}, y_{0}\right)$, let $N\left(x_{0}, y_{0}, E\right)$ denote the number-possibly zero, possibly plus infinity-of points $\left(u_{0}, v_{0}\right)$ in $E$ such that $x\left(u_{0}, v_{0}\right)=x_{0}, y\left(u_{0}, v_{0}\right)=y_{0}$. If one tries to parallel the reasoning used to obtain (6.1), one encounters trouble-the functions $N(x, y, E)$ are generally too large. The following example illustrates the difficulty. Let $\mathbb{C}$ be a Peano curve given by

$$
\mathfrak{E}: x=x(u), y=y(u), z=0, \quad 0 \leqq u, v \leqq 1,
$$

which fills the unit square $0 \leqq x, y \leqq 1$. If $\mathbb{E}$ is regarded as a path surface, and $Q$ denotes the unit square $0 \leqq u, v \leqq 1$, it is clear that $N(x, y, Q)$ is everywhere plus infinity for $0 \leqq x, y \leqq 1$. On the other hand, it is easily seen that the Lebesgue area $L(\mathbb{E})$ is zero (see $\S 3$ ). So there is no hope for a formula for the Lebesgue area analogous to (6.1) for the length of a curve.

8. The essential multiplicity with respect to a region. A study of the works of Geöcze (see Geöcze $[1,2,3,4]$ ) led Radó (see Radó $[2,3])$ to replace the function $N(x, y, E)$ defined in $\$ 7$ by a generally smaller function which may be described as follows. Let $\Re$ be a bounded finitely connected Jordan region, ${ }^{9}$ and let $x(u, v), y(u, v)$ be

${ }^{\circ} \mathrm{A}$ region is a connected open set plus its boundary. A bounded finitely connected Jordan region is a bounded region whose boundary consists of a finite number of simple closed curves. 
two functions which are defined, single-valued, real-valued, and continuous on $\Re$. These determine a continuous transformation $T$ from $\Re$ in the $u v$-plane into the $x y$-plane,

$$
T: x=x(u, v), y=y(u, v), \quad(u, v) \in \Re .
$$

Observe that $T$ may be regarded as a representation for a flat continuous surface - that is, a continuous surface lying in the $x y$-plane (see $\S 2)$. For any set $E$ in $\Re$, the function $N(x, y, E)$ defined in $\$ 7$ will be more completely denoted by $N(x, y, T, E)$-it is known as the crude multiplicity of the point $(x, y)$ under the transformation $T$ with respect to the set $E$ (see Radó and Reichelderfer [1], Radó [4, IV. 1]). If $T_{*}$ be any other continuous transformation defined on $\Re$,

$$
T_{*}: \quad x=x_{*}(u, v), y=y_{*}(u, v), \quad(u, v) \in \Re,
$$

then the distance between $T$ and $T_{*}$ on $\Re$ is the least upper bound of the distances between the points $[x(u, v), y(u, v)]$ and $\left[x_{*}(u, v), y_{*}(u, v)\right]$ for $(u, v)$ in $\Re$. If $\left(x_{0}, y_{0}\right)$ be any point in the $x y$-plane, the essential multiplicity $\kappa\left(x_{0}, y_{0}, T, \Re\right)$ of $\left(x_{0}, y_{0}\right)$ under $T$ with respect to $\Re$ is defined as follows. $\kappa\left(x_{0}, y_{0}, T, \Re\right)$ has the non-negative integral value $k$ if (i) all transformations $T_{*}$ sufficiently close to $T$ have a crude multiplicity $N\left(x_{0}, y_{0}, T_{*}, \Re\right)$ not less than $k$, but (ii) there are continuous transformations $T_{\#}$ arbitrarily close to $T$ for which the crude multiplicity $N\left(x_{0}, y_{0}, T_{\sharp}, \Re\right)$ is exactly $k . \kappa\left(x_{0}, y_{0}, T, \Re\right)$ is set equal to plus infinity if for every positive integer $k$ all transformations $T_{*}$ sufficiently close to $T$ have a crude multiplicity $N\left(x_{0}, y_{0}, T_{*}, \Re\right)$ not less than $k$ (see Radó and Reichelderfer [1], Reichelderfer [2]). Clearly the essential multiplicity $\kappa(x, y, T, \Re)$ never exceeds the crude multiplicity $N(x, y, T, \Re)$-in fact, ${ }^{10}$ if $\Re^{0}$ denotes the set of interior points of $\Re$ then $\kappa(x, y, T, \Re)$ cannot exceed $N\left(x, y, T, \Re^{0}\right)$. The essential multiplicity $\kappa(x, y, T, \Re)$ is a lower semi-continuous function of $(x, y)$ - given any sequence of points $\left(x_{n}, y_{n}\right)$ converging to a point $\left(x_{0}, y_{0}\right)$, it follows that $\lim \inf \kappa\left(x_{n}, y_{n}, T, \Re\right)$ is not less than $\kappa\left(x_{0}, y_{0}, T, \Re\right)$. The essential multiplicity $\kappa(x, y, T, \Re)$ is a lower semi-continuous functional of $T$-given any sequence of continuous transformations $T_{n}$ defined on $\Re$ and converging to a continuous transformation $T_{0}$ on $\Re$, it follows that $\lim \inf \kappa\left(x, y, T_{n}, \Re\right)$ is not less than $\kappa\left(x, y, T_{0}, \Re\right)$.

9. An intrinsic characterization for the essential multiplicity. If the essential multiplicity $\kappa(x, y, T, \Re)$ defined in $\S 8$ is to be used to solve

${ }^{10}$ For a discussion and proof of the properties of the essential multiplicity, see Radó and Reichelderfer [1], or Rad6 [4, IV. 1]. 
the area problem (see $\$ 4$ ), it is clear that one must find a characterization-that is, an equivalent definition-for $\kappa(x, y, T, \Re)$ in terms of the continuous transformation $T$ alone (see (8.1)). Such an intrinsic characterization has been found (see Radó and Reichelderfer [1], Radó $[4, I V .1])$, and will be described presently. If $C$ be any simple closed curve in $\Re$ its image $\bar{C}$ under $T$ is a continuous closed curve in the $x y$-plane. Now if $\left(x_{0}, y_{0}\right)$ be any point not on $\bar{C}$, the angle which the vector with end points $\left(x_{0}, y_{0}\right)$ and $[x(u, v), y(u, v)],(u, v) \in C$, makes with a fixed direction has an algebraic change of the form $2 \pi n$ as $(u, v)$ traverses $C$ once, where $n$ is an integer depending only upon $\left(x_{0}, y_{0}\right), T, C$, and the direction in which $C$ is traversed. Let $\mathfrak{r}$ be any bounded finitely connected Jordan region in $\Re$; it is bounded by a finite number of simple closed curves $C_{0}, C_{1}, \cdots, C_{m}$ (see §8); denote by $\bar{C}_{0}, \bar{C}_{1}, \cdots, \bar{C}_{m}$ the respective images of these curves under $T$. Let $\left(x_{0}, y_{0}\right)$ be any point of the $x y$-plane not on any one of the continuous curves $\bar{C}_{0}, \bar{C}_{1}, \cdots, \bar{C}_{m}$, and let $n_{i}$ denote the integer associated with $\left(x_{0}, y_{0}\right), T, C_{i}$ as $(u, v)$ traverses $C_{i}$ in the positive sense relative to $\mathfrak{r}$, in the manner just described. The index $\mu\left(x_{0}, y_{0}, T, \mathfrak{x}\right)$ is defined as the sum $n_{0}+n_{1}+\cdots+n_{m}$ (see Radó [4, II. 4]). Given any point $\left(x_{0}, y_{0}\right)$ in the $x y$-plane, let $T^{-1}\left(x_{0}, y_{0}\right)$ denote the set of all points $\left(u_{0}, v_{0}\right)$ in $\mathfrak{r}$ for which $x\left(u_{0}, y_{0}\right)=x_{0}, y\left(u_{0}, v_{0}\right)=y_{0}$. The continuity of the transformation $T$ insures that $T^{-1}\left(z_{0}, y_{0}\right)$ is a closed set, which may be empty. If $T^{-1}\left(x_{0}, y_{0}\right)$ is not empty, let $\sigma$ denote any component-a maximal connected subset-of $T^{-1}\left(x_{0}, y_{0}\right)$. Then $\sigma$ is termed essential if in every neighborhood of $\sigma$ in $\Re$ there exists a finitely connected Jordan region $\mathfrak{r}$ containing $\sigma$ in its interior and such that $\left(x_{0}, y_{0}\right)$ does not lie on the image under $T$ of any one of the simple closed curves bounding $\mathfrak{r}$ and the index $\mu\left(x_{0}, y_{0}, T, \mathfrak{r}\right)$ is not zero; otherwise $\sigma$ is not essential. Observe that the property of being an essential component of $T^{-1}\left(x_{0}, y_{0}\right)$ is a local property-if $T_{*}$ is any continuous transformation defined on $\Re$ which is identical with $T$ in a neighborhood of $\sigma$, then $\sigma$ is also a component of $T_{*}^{-1}\left(x_{0}, y_{0}\right)$, and $\sigma$ is an essential component of $T_{*}^{-1}\left(x_{0}, y_{0}\right)$ if and only if $\sigma$ is an essential component of $T^{-1}\left(x_{0}, y_{0}\right)$. It is proven that for any point $\left(x_{0}, y_{0}\right)$ in the $x y$-plane, the essential multiplicity $\kappa\left(x_{0}, y_{0}, T, \Re\right)$ is equal to the number of components of the set $T^{-1}\left(x_{0}, y_{0}\right)$ which are essential (see Radó and Reichelderfer [1]; Radó [4, IV. 1]). Thus $\kappa(x, y, T, \Re)$ may be defined in terms of the transformation $T$ alone.

10. The essential multiplicity with respect to a domain. In order to use the essential multiplicity to effect a solution of the area problem, it seems necessary to have the concept defined when the continuous transformation is defined over a domain-a connected open 
set. Let $\mathcal{D}$ be any bounded domain in the $u v$-plane, and let $x(u, v)$, $y(u, v)$ be two functions which are defined, single-valued, real-valued, bounded, and continuous on $\mathcal{D}$-nothing is assumed about the behavior of these functions, or even their definition, on the boundary of $D$. These determine a bounded continuous transformation $T$ from $\mathcal{D}$ in the $u v$-plane into the $x y$-plane,

$$
T: x=x(u, v), y=y(u, v), \quad(u, v) \in \mathcal{D} .
$$

Now there are sequences of finitely connected Jordan regions $\Re_{n}$ in $\mathcal{D}$ such that any given closed set in $\mathcal{D}$ is contained in $\Re_{n}$ for all $n$ sufficiently large-such sequences are said to fill up $\mathcal{D}$ from the interior (see Radó and Reichelderfer [1]). For each value of $n$, consider the continuous transformation

$$
T_{n}: \quad x=x(u, v), y=y(u, v), \quad(u, v) \in \Re_{n} .
$$

For each point $\left(x_{0}, y_{0}\right)$ in the $x y$-plane the essential multiplicity $\kappa\left(x_{0}, y_{0}, T_{n}, \Re_{n}\right)$ has been defined in $\S 8$. As $n$ tends to infinity, it is shown that the numbers $\kappa\left(x_{0}, y_{0}, T_{n}, \Re_{n}\right)$ have a limit $k$-this limit being either a non-negative integer or plus infinity. Clearly $k$ is independent of the choice of the sequence of Jordan regions $\Re_{n}$ which fill up $\mathcal{D}$ from the interior. The essential multiplicity $\kappa\left(x_{0}, y_{0}, T, \mathcal{D}\right)$ of $\left(x_{0}, y_{0}\right)$ under $T$ with respect to $\mathcal{D}$ is defined to have this value $k$. From the intrinsic characterization of $\kappa\left(x_{0}, y_{0}, T_{n}, \Re_{n}\right)$ described in $\$ 9$, it follows easily that $\kappa\left(x_{0}, y_{0}, T, \mathcal{D}\right)$ is equal to the number of components of the $\operatorname{set}^{11} T^{-1}\left(x_{0}, y_{0}\right)$ which are essential (see Radó and Reichelderfer [1]; Radó [4, IV. 1]). Thus $\kappa(x, y, T, \mathcal{D})$ may be defined in terms of the transformation $T$ alone. Further, $\kappa(x, y, T, D)$ is lower semicontinuous with respect to both $(x, y)$ and $T$ (cf. $\S 8$ ).

11. A comparison of the definitions for the essential multiplicity* If one compares the definition of the essential multiplicity $\kappa(x, y, T, \mathcal{D})$ when the range of $T$ is a domain (see $\$ 10$ ) with the definition of the essential multiplicity $\kappa(x, y, T, \Re)$ when the range of $T$ is a Jordan region (see $\$ 8$ ), a question naturally arises. It is clear that the definition for $\kappa(x, y, T, \mathcal{D})$ might have been made, without using the definition for the essential multiplicity with respect to Jordan regions, in a way completely analogous to that for $\kappa(x, y, T, \Re)$ in $\S 8$. Are these two possible definitions equivalent, or are two basically different ideas involved here? Let $T$ be a bounded continuous transformation as in (10.1), and let $\left(x_{0}, y_{0}\right)$ be any point in the $x y$-plane. If $k$ be any non-

11 As in $\$ 9, T^{-1}\left(x_{0}, y_{0}\right)$ denotes the set of all points $\left(u_{0}, v_{0}\right)$ in $\mathcal{D}$ such that $x\left(u_{0}, v_{0}\right)=x_{0}, y\left(u_{0}, v_{0}\right)=y_{0}$. 
negative integer not greater than $\kappa\left(x_{0}, y_{0}, T, D\right)$, it is clear from the definitions of the essential multiplicities in $\$ \$ 8$ and 10 that all continuous transformations $T_{*}$ sufficiently close to $T$ on $\mathcal{D}$ have a crude multiplicity $N\left(x_{0}, y_{0}, T_{*}, \mathcal{D}\right)$ not less than $k$. But if $\kappa\left(x_{0}, y_{0}, T, \mathcal{D}\right)$ has the value $k$, do there exist continuous transformations $T_{\#}$ defined on $\mathcal{D}$ and arbitrarily close to $T$ on $\mathcal{D}$ for which the crude multiplicity $N\left(x_{0}, y_{0}, T_{\#}, \mathcal{D}\right)$ is exactly $k$ ? In view of what one is trying to donamely, to replace the crude multiplicity of a point $\left(x_{0}, y_{0}\right)$ under a continuous transformation $T$ by a generally smaller multiplicity which has significance (cf. $\$ \$ 7,8$ ), this question gains importance. It has recently been answered in the affirmative (see Reichelderfer [2]) - given an arbitrary positive number $\epsilon$, the continuous transformation $T$ has been modified to yield a continuous transformation $T_{\text {\# }}$ defined on $\mathcal{D}$ whose distance from $T$ on $\mathcal{D}$ is less than $\epsilon$, and for which the crude multiplicity $N\left(x_{0}, y_{0}, T_{\#}, \mathcal{D}\right)$ is equal to the essential multiplicity $\kappa\left(x_{0}, y_{0}, T, \mathcal{D}\right)$. Thus the definition for the essential multiplicities as given in $\$ \$ 8$ and 10 , though differently phrased, are basically the same.

12. A measure for the essential part of a surface. Again, let $T$ be a continuous transformation defined over a bounded finitely connected Jordan region $\Re$ (see $\S 8$ ). Let $\mathcal{D}$ be any domain in $\Re$, and consider the bounded continuous transformation (cf. \$10)

$$
T(\mathcal{D}): \quad x=x(u, v), y=y(u, v), \quad(u, v) \in \mathcal{D} .
$$

For each point $\left(x_{0}, y_{0}\right)$ in the $x y$-plane, $\kappa\left(x_{0}, y_{0}, T,(\mathcal{D}), \mathcal{D}\right)$-denote it simply by $\kappa\left(x_{0}, y_{0}, T, \mathcal{D}\right)$-is equal to the number of components of $T^{-1}\left(x_{0}, y_{0}\right)$ which lie in $\mathcal{D}$ and are essential (see $\left.\$ 10\right)$. Now $\kappa(x, y, T, \mathcal{D})$ is a non-negative measurable function of $(x, y)$ which is zero outside of a certain rectangle, and ${ }^{12} \iint \kappa(x, y, T, \mathcal{D})$ may be regarded as a measure for the essential part of the flat continuous surface represented by $(12.1)$ (see $\$ \$ 6,8)$.

13. A partial solution to the area problem. Now consider a continuous path surface $S$ given by the representation (2.1), and suppose the Lebesgue measure $L(S)$ of $S$ is finite (see $\$ 3$ ). Under these hypotheses, an expression for $L(S)$ analogous to that for the length of a curve given in (6.2) is now described. Let

${ }^{12}$ Let $R$ be any rectangle in the $x y$-plane such that $\kappa(x, y, T, \mathcal{D})$ is zero for $(x, y)$ not in $R$. If $\kappa(x, y, T, \mathcal{D})$ is summable on $R$, then $\iint_{\kappa}(x, y, T, \mathcal{D})$ denotes the Lebesgue integral of $\kappa(x, y, T, D)$ on $R$; if $\kappa(x, y, T, \mathcal{D})$ is not summable on $R$, then $\iint_{\kappa}(x, y, T, \mathcal{D})$ is defined to be plus infinity. Clearly $\iint_{\kappa}(x, y, T, \mathcal{D})$ is independent of the choice of $R$, so long as $\kappa(x, y, T, D)$ is zero for $(x, y)$ not in $R$. 


$$
\begin{aligned}
& T_{x}: \quad y=y(u, v), \quad z=z(u, v), \quad 0 \leqq u, v \leqq 1 \text {; } \\
& T_{y}: \quad z=z(u, v), \quad x=x(u, v), \quad 0 \leqq u, v \leqq 1 \text {; } \\
& T_{z}: \quad x=x(u, v), y=y(u, v), \quad 0 \leqq u, v \leqq 1 \text {, }
\end{aligned}
$$

be representations for the flat continuous surfaces obtained by projecting the representation for $S$ upon the coordinate planes $y z, z x, x y$ respectively. If $K$ be the generic notation for a finite system of disjoint domains $\mathcal{D}$ in $0 \leqq u, v \leqq 1$, then ${ }^{13}$

$$
\begin{aligned}
L(S)= & \text { l.u.b. } \sum_{\mathcal{D} \in K}\left\{\left[\iint \kappa\left(y, z, T_{x}, \mathcal{D}\right)\right]^{2}\right. \\
+ & {\left[\iint \kappa\left(z, x, T_{y}, \mathcal{D}\right)\right]^{2} } \\
+ & {\left.\left[\iint \kappa\left(x, y, T_{z}, \mathcal{D}\right)\right]^{2}\right\}^{1 / 2}, }
\end{aligned}
$$

the least upper bound being taken with respect to all finite systems $K$ of disjoint domains in $0 \leqq u, v \leqq 1$. Observe that the essential multiplicity functions described in $\$ \$ 8-10$ play the role of the crude multiplicity functions in $\$ 6$. It is not yet possible to describe a sequence of finite systems $K_{n}$ of disjoint domains such that $L(S)$ is the limit of sums of the above radical over the $K_{n}$-that is, so that (13.1) may be replaced by a formula analogous to (6.1)-although such a sequence of $K_{n}$ must exist. Nor is it possible to assert that the expression in the right member of (13.1) must be plus infinity if the Lebesgue area $L(S)$ is plus infinity, although it has been shown that the expression in the right member of (13.1) cannot then be zero. ${ }^{14}$

\section{BIBLIOGRAPHY}

\section{S. BANACH}

1. Sur les lignes rectifiables et les surfaces dont l'aire est finie, Fund. Math. vol. 7 (1925) pp. 225-236.

Z. DE GEÖCZE

1. Quadrature des surfaces courbes, Mathematische und naturwissenschaftliche Berichte aus Ungarn vol. 26 (1908) pp. 1-88.

2. Recherches gênérales sur la quadrature des surfaces courbes, First memoir, Mathe-

${ }^{13}$ See Rado [4, V. 2] for an extensive discussion of this partial solution to the area problem.

${ }^{14}$ Since this address was delivered, Professor Rado has informed the writer that by using certain results of Cesari-see L. Cesari, Carratterizzazione analitica delle superficie continue di area finita secondo Lebesgue, Annali R. Scuola Normale Superiore Pisa (2) vol. 10 (1941) pp. 253-294 and vol. 11 (1942) pp. 1-42-it is now possible to establish (13.1) even if the Lebesgue area $L(S)$ is plus infinity. 
matische und naturwissenschaftliche Berichte aus Ungarn vol. 27 (1909) pp. 1-21.

3. Recherches générales sur la quadrature des surfaces courbes, Second memoir, Mathematische und Naturwissenschaftliche Berichte aus Ungarn vol. 27 (1909) pp. 131-163.

4. Recherches gênérales sur la quadrature des surfaces courbes, Third memoir, Mathematische und Naturwissenschaftliche Berichte aus Ungarn vol. 30 (1912) pp. 1-29.

H. LEBESGUE

1. Intégrale, longueur, aire, Annali di Matematica pura ed applicata vol. 7 (1902) pp. 231-359.

T. RADó

1. Sur l'aire des surfaces courbes, Acta Univ. Szeged. vol. 3 (1927) pp. 131-169.

2. Über das Flächenmass rektifizierbarer Flächen, Math. Ann. vol. 100 (1928) pp. 445-479.

3. On absolutely continuous transformations in the plane, Duke Math. J. vol. 4 (1938) pp. 189-221.

4. Length and area, Amer. Math. Soc. Colloquium Publications, vol. 30, not yet published.

T. RADó and P. REICHELDERFER

1. A theory of absolutely continuous transformations in the plane, Trans. Amer. Math. Soc. vol. 49 (1941) pp. 258-307.

P. V. REICHELDERFER

1. On bounded variation and absolute continuity for parametric representations of continuous surfaces, Trans. Amer. Math. Soc. vol. 53 (1943) pp. 251-291.

2. On the definition of the essential multiplicity for continuous transformations in the plane, Trans. Amer. Math. Soc. vol. 62 (1947) pp. 284-314.

H. A. SCHWARZ

1. Sur une définition erronée de l'aire d'une surface courbe, Gesammelte Mathematische Abhandlungen von H. A. Schwarz, vol. 2, 1890, pp. 309-311.

UNIVERSITY OF WISCONSIN 